\title{
Relação entre as potências de sprint e salto vertical em jovens atletas de futebol
}

\author{
Relationship between the sprint and vertical jumps' power in young \\ soccer players
}

\author{
C.J. Silva-Junior, A. Palma, P. Costa, P.P. Pereira-Junior, R.C.L. Barroso, R.C. Abrantes- \\ Junior, M.A.M. Barbosa
}

RESUMO

A potência muscular, a velocidade e a aceleração são constantemente citadas como importantes e determinantes para alcançar um alto nível de performance nos desportos em geral. Desta forma, o presente estudo teve por objetivo examinar as correlações existentes entre as potências de sprints de 10 e 30 metros e a potência de salto vertical em jovens atletas de futebol. Participaram do estudo 143 jogadores de futebol do sexo masculino das categorias Sub-20 $(n=34)$, Sub-17 $(n=43)$ e Sub-15 $(n=$ 66 ), os quais realizaram testes de salto vertical, velocidade de 10 metros e velocidade de 30 metros. Os resultados apontaram desde correlações baixas a moderadas quando comparados os valores absolutos de tempo e altura alcançada $(r=-.47$ a .71). Ao se comparar os valores de potência obtidos nos testes, foram encontradas desde correlações moderadas a altas $(r=.74$ a .87). Os resultados sugerem que a realização destes três testes é importante pois mensuraram capacidades motoras distintas. Além disso, a correlação entre a potência de salto vertical e de sprint de 30 metros é surpreendentemente forte. Contudo, novos estudos com a mesma proposta devem ser conduzidos para que haja um melhor entendimento sobre estas relações de potência.

Palavras-chave: futebol, potência muscular, velocidade, jovens atletas

ABSTRACT

Muscle power, speed and acceleration are generally considered important determinants of high performance in sports. Thus, the aim of this study was to evaluate the relationships between 10- and 30-meter sprint power and vertical jump power among young soccer players. The participants of the study were 143 male soccer players from the categories Under-20 $(n=34)$, Under-17 $(n=43)$ and Under-15 $(n=66)$, which performed vertical jump test, 10-meter sprint test and 30-meter sprint test. The results indicated weak to moderate correlations when the absolute values of time and maximal height were compared ( $r=-.47$ to .71$)$. When the values of power obtained from tests were compared, moderate to strong correlations were found ( $r=.74$ to .87$)$. The results suggest that implementing these three tests would be important because they measure different motor skills. Moreover, the correlation between vertical jump power and 30-meter sprint power was surprisingly strong. Nevertheless, further studies are required in order to improve understanding about these power relationships.

Keywords: soccer, muscle power, speed, young athletes

Submetido: 28.06.2010 | Aceite: 16.11.2010

Celso José da Silva-Junior, Alexandre Palma, Paulo Costa e Pedro Paulo Pereira-Junior. Escola de Educação Física e Desportos, Universidade Federal do Rio de Janeiro, Brasil.

Rafael de Castro Lima Barroso. Escola de Educação Física e Desportos, Universidade Federal do Rio de Janeiro; Fluminense Football Club, Rio de Janeiro, Brasil.

Ricardo Costa Abrantes-Junior e Márcio Assis Marques Barbosa. Fluminense Football Club, Rio de Janeiro, Brasil. Endereço para correspondência: Celso José da Silva Junior, Rua Muiatuca 220/108, CEP: 21921-680 - Rio de Janeiro / RJ, Brasil.

E-mail: celsinhosilva@hotmail.com 
A força e potência muscular são características fisiológicas importantes na prática do futebol (Hoff, 2005) e o desenvolvimento destas estaria intimamente ligado ao sucesso no alto nível do referido desporto (Brodt, Wagner, \& Heath, 2008; Secora, Latin, Berg, \& Noble, 2004). Apesar do futebol se tratar de uma atividade de longa duração, os momentos decisivos do jogo em sua maioria ocorrem em lances de alta intensidade, curtas distâncias e durações. Barros, Valquer e Sant'anna (1999) demonstraram que $96 \%$ dos sprints realizados durante um jogo de futebol são inferiores a 30 metros, sendo $49 \%$ em distâncias menores que 10 metros. Sendo assim, Rebelo e Oliveira (2006) afirmam que ações importantes do jogo estão ligadas a uma produção elevada de potência a nível muscular. Podem ser destacadas ações como saltos, deslocamentos em velocidade de curtas distâncias, dribles e todo tipo de movimentação tática do jogo. Seguindo esta linha, Harris, Stone, O’Bryant, Proulx e Johnson (2000) afirmam que o sucesso em vários esportes depende da capacidade que o atleta tem de dominar uma variedade de habilidades que estão relacionadas a níveis de força, potência e velocidade.

Peterson, Alvar e Rhea (2006) afirmam que a potência muscular está relacionada à capacidade de gerar força aliada a uma alta velocidade no movimento. Assim, a potência muscular é vista como uma importante variável para testes físicos e treinamentos, especialmente para esportes que envolvem velocidade e saltos. Corroborando esta idéia, Markovic, Jukic, Milanovic e Metikos (2007) observam resultados importantes quanto ao treinamento de potência. O estudo aponta que o treinamento de velocidade e pliometria promove ganhos de performance em potência muscular após 10 semanas de treinamento, sugerindo que estes tipos de treino são importantes para a melhora de potência muscular em atletas em geral.

Atualmente, é notório que o conhecimento científico está incorporado com um maior grau de importância no planejamento e execução do treinamento, fato este que é bem destacado por Bangsbo, Mohr e Krustrup (2006). Treinadores físicos e cientistas do esporte usam testes físicos de campo para mensurar a performance atual dos seus atletas devido ao alto custo de equipamentos laboratoriais. Corroborando esta idéia, Duarte, Fiúza e Pereira (2005) afirmam que os métodos de avaliação direta implicam na utilização de instrumentos que não estão ao dispor de todos os treinadores, sendo assim importante encontrar outros métodos mais acessíveis, tais como os testes de campo. Além disso, Vescovi e McGuigan (2008) afirmam que para a realização de testes físicos é necessária a familiarização com as técnicas dos movimentos, pois o repertório motor do atleta pode comprometer a acurácia dos métodos assim como a segurança durante a avaliação.

A literatura atual apresenta um número escasso de trabalhos que procuraram relacionar velocidade, aceleração e a potência em saltos verticais apesar de tais capacidades serem comummente observadas em um desporto como o futebol. Little e Williams (2005) relataram que a aceleração (tempo obtido no sprint de $10 \mathrm{~m}$ ), velocidade máxima (tempo obtido no sprint de $20 \mathrm{~m}$ lançados) e agilidade são características motoras distintas em um grupo de jogadores profissionais de futebol, sugerindo assim que as características motoras citadas devem ser testadas de forma específica. Em estudo realizado com atletas universitárias de voleibol, Barnes et al. (2007) indicam que indivíduos com grande performance na altura alcançada no salto com contra-movimento (CMJ) têm baixos tempos em testes de agilidade, sugerindo que o treinamento de saltos verticais poderia apresentar melhoras em determinados tipos de agilidade. Vescovi e McGuigan (2008) encontraram correlações baixas entre a altura alcançada no CMJ e os tempos obtidos nos testes de aceleração de 9.1 metros $(-.49,-.65$ e -.68$)$ para grupos de atletas do sexo feminino de futebol colegial, futebol universitário e lacrosse universitário, respectivamente. 
Como pode ser visto, os estudos ainda mostram certa divergência com relação aos resultados. Além disso, na literatura são encontrados poucos trabalhos que propõem correlações entre as potências de saltos verticais e sprints. Em grande maioria são analisados os resultados absolutos de tempo obtido no sprint e altura máxima alcançada no salto. Desta forma, o presente estudo tem por objetivo examinar as correlações existentes entre as potências de sprints de 10 e 30 metros e a potência de salto vertical em jovens atletas de futebol.

\section{Amostra}

\section{MÉTODO}

Participaram do estudo 143 jogadores de futebol do sexo masculino das categorias de base do Fluminense Football Club devidamente registrados na Federação de Futebol do Estado do Rio de Janeiro, instituição filiada à Confederação Brasileira de Futebol. Entre os atletas, 34 pertenciam à equipe Sub-20 (isto é, tinham entre 18 e 20 anos de idade), 43 atletas da equipe Sub-17 (idades entre 16 e 17 anos) e 66 atletas da equipe Sub-15 (idades entre 14 e 15 anos). Todos os atletas que realizaram os testes físicos tinham no mínimo dois anos de experiência no treinamento de futebol e estavam livres de lesões que poderiam prejudicar a máxima performance nas avaliações. Todos os atletas receberam cargas de treinos entre 15 e 20 horas semanais (distribuídos entre treinos físicos, técnicos e táticos), além da participação em jogos oficiais no mínimo uma vez na semana. Os participantes do estudo estavam inseridos no mais alto nível competitivo das suas respectivas categorias, representando o clube nas competições mais importantes tanto ao nível estadual, quanto nacional.

Estes testes fazem parte da rotina de avaliações à qual os atletas são submetidos. Tais avaliações têm periodicidade quadrimestral e os protocolos posteriormente citados são aplicados em todas as categorias há no mínimo dois anos, garantindo assim a familiarização tanto dos atletas quanto dos avaliadores com os testes propostos. Os dados, então, foram armazenados em um banco para posterior uso pela Comissão Técnica. Ressaltese, portanto, que os dados, embora coletados com o máximo de rigor, não foram aferidos especificamente para $o$ presente estudo. Contudo, os atletas foram notificados que poderiam desistir de participar dos testes a qualquer momento e todos concordaram de modo voluntário. Para utilização do banco de dados, por outro lado, os pesquisadores informaram ao clube o objetivo $\mathrm{e}$ os procedimentos da pesquisa, bem como, garantiram o caráter sigiloso dos elementos individuais.

\section{Instrumentos}

A altura alcançada no CMJ foi obtida através do uso de um tapete de contato (Axon Jump V2.01, Argentina) que possui um cronômetro com precisão em milésimos de segundo, sendo que a altura alcançada é calculada através das fórmulas da física clássica, conhecendo-se a aceleração da gravidade local e o tempo de vôo do salto. Para a mensuração dos sprints de 10 e 30 metros foi usado um equipamento de células fotoelétricas (Microgate Racetime2, Itália). Na avaliação antropométrica foi usado um estadiômetro Cardiomed para medir a estatura e uma balança mecânica Filizola para quantificar a massa corporal.

\section{Procedimentos}

A realização dos testes físicos foi dividida em dois dias, sendo que no primeiro foi realizado o salto vertical posteriormente à avaliação antropométrica e no segundo os sprints de 10 metros e 30 metros. Na realização do CMJ os participantes estavam na posição inicial de pé e mãos situadas na cintura, em seguida realizavam um agachamento com flexão de joelhos até $90^{\circ}$ com imediato salto de máxima altura. As mãos devem permanecer na cintura durante todo o 
movimento com o objetivo de eliminar qualquer influência de movimento dos braços, pois o uso dos mesmos em um salto vertical promove ganhos na performance quando comparado à realização do mesmo salto sem o uso destes (Mourão \& Gonçalves, 2008). Além disso, os participantes devem manter quadris e joelhos completamente estendidos na fase aérea do salto. Cada atleta teve direito à realização de três saltos com intervalo de descanso de no mínimo 30 segundos entre cada salto. Caso algum desses procedimentos não fosse respeitado, o atleta faria outra repetição da realização do $\mathrm{CMJ}$ após um período de descanso. Antes da realização do teste, foi explicada e demonstrada aos atletas a técnica de salto, sendo permitido a cada participante a realização de dois saltos submáximos. O motivo da escolha do referido protocolo é o fato deste permitir discriminar a contribuição dos membros inferiores no desempenho do salto (Markovic, Dizdar, Jucik, \& Cardinale, 2004).

Na realização dos sprints de 10 e 30 metros, foram usados dois pares de fotocélulas com altura de 1 metro, sendo um par situado na linha inicial e o outro par na linha final a 10 metros e posteriormente a 30 metros. Cada atleta teve direito à realização de dois sprints de 10 metros e dois de 30 metros. Os participantes começavam em posição de pé na linha inicial e eram instruídos a correr em máxima velocidade até ultrapassar o par de fotocélulas situado na linha final. Antes da realização do teste, os atletas foram submetidos a um protocolo de aquecimento com trote, sprints curtos e movimentos com mudança de direção, totalizando 10 minutos.

Para o cálculo de potência de saltos verticais foi usado o modelo proposto por Sayers, Harackiewicz e Harman (1999) onde o pico de potência (PP) é descrito como: PP (Watts) = $60.7 \times$ (altura do salto vertical) $+45.3 \times$ (massa corporal) - 2055, sendo a altura do salto vertical dada em centímetros e a massa corporal em quilos. O modelo usado é bem descrito por Peterson et al. (2006), onde os autores observaram as correlações existentes entre potência de membros inferiores e resultados de testes físicos de alta intensidade e curta duração.

A potência dos sprints de 10 e 30 metros foi obtida através do mesmo modelo usado para calcular a potência máxima (PM) de deslocamento no Running-based Anaerobic Sprint Test - RAST (Wolverhampton, Reino Unido), que é descrita como: PM (Watts) = massa corporal $\times(\text { distância percorrida })^{2} /($ tempo do sprint) ${ }^{3}$, sendo a massa corporal dada em quilos, a distância percorrida em metros e o tempo obtido em segundos. O modelo de obtenção da PM é bem descrito e utilizado por Roseguini, Silva e Gobatto (2008) e Pellegrinotti et al. (2008). Em outro estudo utilizando o mesmo modelo de obtenção da PM, Paradisis, Tziortzis, Zacharogiannis, Smirniotou e Karatzanos (2005) obtiveram correlações significativas entre a PM e resultados máximos de provas de 100 metros no atletismo.

\section{Análise Estatística}

Os coeficientes de correlação foram calculados através da correlação de Pearson e usados para determinar a força e direção das relações dentre as variáveis testadas. Além disso, o coeficiente de determinação $\left(r^{2}\right)$ foi usado para examinar o quanto da variação total é comum entre os valores que constituem cada um dos pares analisados. A significância estatística adotada foi de $p<.05$. Todos os dados são apresentados em média e desvio padrão (DP).

\section{RESULTADOS}

Os resultados das avaliações estão expostos na Tabela 1 de acordo com cada categoria e para a amostra geral estudada. Com relação às avaliações antropométricas, podemos observar que as categorias Sub-17 e Sub-20 apresentam valores médios de estatura bem próximos e com o mesmo desvio padrão. Quando observamos os tempos obtidos em ambos os sprints, podemos notar que a categoria Sub-15 
Potência em jovens atletas de futebol | 9

Tabela 1

Valores médios (M) \pm desvio padrão (DP) das avaliações para as categorias Sub-20, Sub-17, Sub-15 e amostra total

\begin{tabular}{lccccccccc}
\hline & \multicolumn{2}{c}{$\begin{array}{c}\text { Sub-15 } \\
(n=66)\end{array}$} & \multicolumn{2}{c}{$\begin{array}{c}\text { Sub-17 } \\
(n=43)\end{array}$} & \multicolumn{2}{c}{$\begin{array}{c}\text { Sub-20 } \\
(n=34)\end{array}$} & \multicolumn{3}{c}{$\begin{array}{c}\text { Amostra Total } \\
(n=143)\end{array}$} \\
& $\mathrm{M}$ & $\mathrm{DP}$ & $\mathrm{M}$ & $\mathrm{DP}$ & $\mathrm{M}$ & $\mathrm{DP}$ & $\mathrm{M}$ & $\mathrm{DP}$ \\
\hline Idade (anos) & 14.25 & .58 & 16.43 & .61 & 18.56 & .85 & 15.93 & 1.86 \\
Estatura (cm) & 169.6 & 8.9 & 175.2 & 6.7 & 175.3 & 6.7 & 173.2 & 8.5 \\
Massa corporal (kg) & 59.5 & 8.9 & 67.6 & 8.5 & 70.2 & 7.8 & 65.0 & 10.0 \\
Tempo 10m (s) & 1.841 & .123 & 1.861 & .092 & 1.745 & .069 & 1.824 & .112 \\
Potência 10m (W) & 982.32 & 257.42 & 1061.22 & 195.48 & 1367.26 & 192.54 & 1097.57 & 272.47 \\
Tempo 30m (s) & 4.507 & .275 & 4.363 & .157 & 4.147 & .122 & 4.378 & .257 \\
Potência 30m (W) & 600.28 & 145.69 & 735.71 & 104.76 & 913.87 & 117.56 & 715.56 & 178.58 \\
Altura CMJ (cm) & 35.6 & 4.3 & 39.0 & 5.0 & 42.6 & 3.9 & 38.3 & 5.2 \\
Potência CMJ (W) & 2802.87 & 469.77 & 3367.00 & 412.79 & 3802.07 & 363.55 & 3212.05 & 592.62 \\
\hline
\end{tabular}

apresenta valores médios mais baixos que a Sub-17. Contudo, ao se analisar a potência dos sprints, nota-se que a categoria Sub-17 apresenta maior valor médio que a Sub-15. É valido ressaltar que em todos os testes realizados a categoria Sub-20 apresentou os melhores valores médios com relação ao índice de desempenho.

A Tabela 2 apresenta os coeficientes de correlação obtidos a partir da correlação de Pearson. Quando comparados somente os valores de tempo e altura alcançada nos respectivos testes, foram encontradas correlações baixas para T10 e HCMJ $(r=$ -.47) e para T30 e HCMJ $(r=-.62)$; moderada para T10 e T30 $(r=.71)$. Contudo, ao se comparar os valores de potência obtidos nos testes, foram encontradas correlações moderadas para P10 e PCMJ $(r=.74)$; altas relações para P10 e P30 $(r=.84)$ e para P30 e PCMJ $(r=.87)$.

A Tabela 3 apresenta os valores obtidos na correlação entre as potências dos sprints e a potência do salto vertical discriminado por categorias e por grupo geral. Todas as correlações obtidas apresentaram significância em $p<.01$.
Tabela 2

Coeficientes de correlação da amostra total de jovens atletas de futebol

\begin{tabular}{crrrrrr}
\hline & T10 & P10 & T30 & P30 & HCMJ & PCMJ \\
\hline T10 & - & & & & & \\
P10 & $-.79^{*}$ & - & & & & \\
T30 & $.71^{*}$ & $-.68^{*}$ & - & & & \\
P30 & $-.52^{*}$ & $.84^{*}$ & $-.82^{*}$ & - & & \\
HCMJ & $-.47^{*}$ & $.43^{*}-.62^{*}$ & $.52^{*}$ & - & \\
PCMJ & $-.32^{*}$ & $.74^{*}-.55^{*}$ & $.87^{*}$ & $.66^{*}$ & - \\
\hline
\end{tabular}

Nota: T10 = Tempo obtido no sprint de 10 metros; P10 = Potência obtida no sprint de 10 metros; T30 = Tempo obtido no sprint de 30 metros; P30 = Potência obtida no sprint de 30 metros; HCMJ = Altura máxima alcançada no $\mathrm{CMJ} ; \mathrm{PCMJ}=$ Potência máxima obtida no CMJ. ${ }^{*} p<.01$

Tabela 3

Correlações entre as potências de sprint de 10 metros, 30 metros e salto vertical

\begin{tabular}{lcccc}
\hline & $\begin{array}{c}\text { Amostra } \\
\text { Total }\end{array}$ & Sub-20 & Sub-17 & Sub-15 \\
\hline P10 × PCMJ & $.74^{*}$ & $.45^{*}$ & $.60^{*}$ & $.71^{*}$ \\
P30 × PCMJ & $.87^{*}$ & $.74^{*}$ & $.78^{*}$ & $.75^{*}$ \\
P10 $\times$ P30 & $.84^{*}$ & $.60^{*}$ & $.70^{*}$ & $.86^{*}$ \\
\hline
\end{tabular}

Nota: T10 = Tempo obtido no sprint de 10 metros; P10 = Potência obtida no sprint de 10 metros; T30 = Tempo obtido no sprint de 30 metros; P30 = Potência obtida no sprint de 30 metros; HCMJ = Altura máxima alcançada no $\mathrm{CMJ} ; \mathrm{PCMJ}=$ Potência máxima obtida no CMJ. ${ }^{*} p<.01$ 


\section{DISCUSSÃO}

Apesar do metabolismo aeróbio ser predominante no fornecimento de energia durante uma partida de futebol, as ações mais decisivas do desporto são nutridas pela via metabólica anaeróbia (Stolen, Chamari, Castagna, \& Wisløff, 2005). Logo, a força muscular e a velocidade são importantes características fisiológicas para atletas de futebol (Reilly, Bangsbo, \& Franks, 2000). O nível de importância das ações de alta intensidade no futebol é descrito por Capranica, Tessitore, Guidetti e Figura (2001) em estudos com jovens atletas de futebol, em que observaram, durante uma partida de futebol, que estímulos de alta intensidade ocorrem em cerca de $62 \%$ do primeiro tempo e $48 \%$ do segundo tempo. Bangsbo (1994) demonstra que jogadores de futebol profissionais realizam em média 1 sprint a cada 90 segundos e 15.5 saltos durante uma partida, ratificando a ideia de que saltos e deslocamentos em velocidade nitidamente influenciam o resultado de um jogo. Sendo assim, frente à importância da potência muscular no referido esporte, o objetivo do presente estudo foi verificar o grau de associação existente entre os testes físicos de potência muscular de membros inferiores em jovens atletas de futebol.

O presente estudo apresenta correlações fracas e moderadas obtidas a partir dos resultados de tempo e altura máxima alcançada nos testes físicos de campo analisados em um grupo de jovens atletas de futebol masculino (Tabela 2). Isto pode sugerir que apesar de os testes avaliarem atributos ou valências físicas similares nenhum dos testes está apto a estimar o desempenho no outro para o grupo estudado. A partir da observação do coeficiente de determinação $\left(r^{2}\right)$ foram obtidos valores entre $22.1 \%$ e $50.4 \%$, sugerindo que as valências testadas são capacidades motoras algo independentes.

Apesar do sprint de 10 metros, de 30 metros e o CMJ serem testes com alta velocidade de execução, foi encontrada correlação baixa entre T10 e HCMJ $(r=-.47)$; e correlações moderadas entre T30 e HCMJ $(r$ $=-.62$ ) para o grupo total avaliado. Em estudo com 17 jogadores de futebol profissional da $1^{\text {a }}$ Divisão da Noruega, Wisløff, Castagna, Helgerud, Jones e Hoff (2004) encontraram correlação moderada entre T10 e HCMJ $(r=.72, p<.001)$, e entre HCMJ e T30 foram encontradas correlações moderadas semelhantes ao observado no presente estudo $(r=.60, p<.01)$.

Em outro estudo com atletas de futebol do sexo feminino, sendo 83 de nível colegial e 51 de nível universitário, Vescovi e McGuigan (2008) apresentam correlações entre o tempo obtido no sprint de 9.1 metros (T9) e HCMJ, assim como correlações entre tempo do sprint de 36.6 metros (T36) e HCMJ. Em nível universitário a correlação T9-HCMJ foi de -.658 e para T36-HCMJ foi obtido -.788. Já para as atletas colegiais foram encontrados resultados para T9-HCMJ e T36-HCMJ de -.491 e -.575 respectivamente, sendo todas as correlações apresentadas com significância de $p<.001$. Seguindo esta linha de raciocínio, o CMJ se mostrou significantemente relacionado $(p<.05)$ aos tempos dos sprints de $30 \mathrm{e}$ 100 metros $(-.60$ e -.64$)$ de 17 atletas de velocidade do sexo feminino de nível colegial (Hennessy \& Kilty, 2001).

Da mesma forma, Cronin e Hansen (2005) demonstraram significantes correlações do CMJ com o tempo de testes de velocidade de 10 e 30 metros ( -.62 e -.56 , respectivamente) em 26 atletas profissionais de rugby do sexo masculino $(p<.05)$. Apesar dos trabalhos citados e o presente estudo apresentarem uma diferença grande entre idade, sexo e esporte praticado pelos sujeitos, surpreendentemente correlações similares foram obtidas entre a altura alcançada no CMJ e o tempo de testes de velocidade. No entanto, indo de encontro aos resultados encontrados na literatura, Marques, Travassos e Almeida (2010) apresentam correlações significantes $(p<.05)$, porém fracas $(-.408)$, entre a altura máxima alcançada em um teste de impulsão vertical e o tempo do 
sprint de 30 metros. É válido ressaltar que o protocolo do salto vertical usado neste estudo não foi o mesmo usado nos diversos estudos citados anteriormente, fato este que pode ter sido determinante na obtenção das correlações.

Quando observamos os resultados obtidos no presente estudo relativos à potência, encontramos desde relações moderadas até fortes. Apesar de não terem sido encontrados na literatura resultados referentes às mesmas correlações obtidas no presente estudo, alguns dados obtidos se mostram conflituantes com os encontrados em outros trabalhos publicados. Ao consultarmos a Tabela 2 podemos observar correlações significantes de -.32 e - .55 para as relações T10-PCMJ e T30PCMJ, respectivamente. Porém em estudo com atletas do sexo masculino de rugby, Cronin e Hansen (2005) encontram resultados de -.11 e .15 para correlações entre potência de salto vertical e tempo no sprint de 10 metros, e entre potência de salto vertical e tempo no sprint de 30 metros, respectivamente.

Em estudo com atletas de levantamento de peso olímpico, Carlock et al. (2004) encontram resultados que indicam uma forte relação entre a potência de salto vertical e a performance no referido esporte. Peterson et al. (2006) encontram correlações significativas $(p<.01)$ de .778 entre potência de salto vertical e a velocidade de um sprint de 36.58 metros em atletas universitários. As correlações encontradas no presente trabalho são apresentadas na Tabela 3. Dentre os estudos citados e o presente estudo, é notório que os resultados encontrados não têm uma conclusão em comum. Além disso, nenhum estudo com a proposta de verificar as relações entre as potências de salto vertical e de sprint foi encontrado.

Pode-se concluir, desta forma, que os resultados obtidos a partir das correlações entre salto vertical e sprints de 10 e 30 metros são importantes à medida que indicam a necessidade da realização de diferentes testes para mensurar capacidades motoras distintas, conforme foi observado nos coeficientes de determinação obtidos. Os testes de campo para mensurar a força dos membros inferiores (CMJ), aceleração (sprint de 10 metros) e velocidade máxima (sprint de 30 metros) devem ser incluídos em uma bateria de testes para atletas de futebol, assim como outras variáveis também devem ser avaliadas, tais como medidas antropométricas, agilidade e capacidade aeróbia e anaeróbia. Além disso, pode ser destacada a forte correlação obtida entre P30-PCMJ (.87, $p<.01)$. Chamari et al. (2004) demonstraram que o tempo em sprints de 30 metros se correlaciona com o pico de velocidade do salto, enquanto o tempo em sprints de 10 metros não apresenta tal relação. $\mathrm{O}$ pico de velocidade de um determinado movimento pode estar influenciando os resultados. Isto poderia explicar a maior correlação encontrada entre P30-PCMJ quando comparada a P10-PCMJ, já que no sprint de 30 metros existe um maior pico de velocidade do que no sprint de 10 metros. Vescovi, Rupf, Brown e Marques (2011) apontam que a maior e menor velocidade média da corrida de 36.6 metros ocorre nos últimos 9.1 metros e nos primeiros 9.1 metros, respectivamente. Contudo, esta forte correlação não indica que exista uma relação de causa e efeito entre as duas variáveis, ainda que estas estejam relacionadas de alguma forma. Ainda, surpreendentemente foram encontradas desde correlações fracas a moderadas $(.45-.74, p<.01)$ entre P10PCMJ. Carvalho e Carvalho (2006) afirmam que duas grandes manifestações de força rápida (força explosiva e potência) estão presentes em todas as situações desportivas nas quais são necessárias alterações rápidas de direção e/ou acelerações. E sabendo que, em sprints de 30 metros, os primeiros 10 metros apresentam um nível de aceleração substancialmente maior do que o restante do percurso (Wisløff et al., 2004), não seria imprudente esperar por relações mais fortes entre P10-PCMJ. No entanto, levando em consideração que a pesquisa possui participantes de três diferentes categorias de base do futebol, é possível que os diferentes regimes de treino (Wisløff et al., 
2004) e estágios de maturação (Hansen, Bangsbo, Twisk, \& Klausen, 1999; Malina, Eisenmann, Cumming, Ribeiro, \& Aroso, 2004; Sherar, Baxter-Jones, Faulkner, \& Russel, 2007) possam exercer influência nos resultados.

Novos estudos com a mesma proposta devem ser conduzidos para que haja um melhor entendimento sobre estas relações de potência, principalmente se novas pesquisas forem aplicadas em atletas com características distintas às dos atletas do presente estudo, como por exemplo: sexo feminino, outras modalidades esportivas, idade mais avançada e diferentes níveis de treinamento. Assim, a partir de novos estudos poderemos ter conclusões mais precisas sobre as possíveis relações da potência e o desempenho em atletas de alto nível.

\section{REFERÊNCIAS}

Bangsbo, J. (1994). The physiology of soccer: With special reference to intense intermittent exercise. Acta Physiologica Scandinavica, 151, 1156.

Bangsbo, J., Mohr, M., \& Krustrup, P. (2006). Physical and metabolic demands of training and match-play in the elite football player. Journal of Sports Sciences, 24(7), 665-674.

Barnes, J. L., Schilling, B. K., Falvo, M. J., Weiss, L. W., Creasy, A. K., \& Fry, A. C. (2007). Relationship of jumping and agility performance in female volleyball athletes. Journal of Strength and Conditioning Research, 21 (4), 11921196.

Barros, T. L., Valquer, W., \& Sant'anna, M. (1999). High intensity motion pattern analysis of Brazilian elite soccer players in different positional roles. Medicine $\mathcal{E}$ Science in Sports $\mathcal{E}$ Exercise, 31 (S5), 260.

Brodt, V., Wagner, D. R., \& Heath, E. M. (2008). Countermovement vertical jump with drop step is higher than without in collegiate football players. Journal of Strength and Conditioning Research, 22 (4), 1382-1385.

Capranica, L., Tessitore, A., Guidetti, L., \& Figura, F. (2001). Heart rate and match analysis in prepubescent soccer players. Journal of Sports Sciences, 19(6), 379-384.
Carlock, J. M., Smith, S. L., Hartman, M. J., Morris, R. T., Ciroslan, D. A., Pierce, K. C., ... Stone, M.H. (2004). The relationship between vertical jump power estimates and weightlifting ability: A field-test approach. Journal of Strength and Conditioning Research, 18(3), 534-539.

Carvalho, C., \& Carvalho, A. (2006). Não se deve identificar força explosiva com potência muscular, ainda que existam algumas relações entre ambas. Revista Portuguesa de Ciências do Desporto, 6(2), 241-248.

Chamari, K., Hachana, Y., Ahmed, Y. B., Galy, O., Sghaïer, F., Chatard, J. C., ... Wisløff, U. (2004). Field and laboratory testing in young elite soccer players. British Journal of Sports Medicine, 38(2), 191-196.

Cronin, J. B., \& Hansen, K. T. (2005). Strength and power predictors of sports speed. Journal of Strength and Conditioning Research, 19(2), 349357.

Duarte, R. F., Fiúza, T. M., \& Pereira, F. A. (2005). Determinação indirecta do máximo $\mathrm{VO}_{2}$ com base no teste de Cooper em jogadores de Futebol. Motricidade, 1(4), 239-245.

Hansen, L., Bangsbo, J., Twisk, J., \& Klausen, K. (1999). Development of muscle strength in relation to training level and testosterone in young male soccer players. Journal of Applied Physiology, 87, 1141-1147.

Harris, G. R., Stone, M. H., O'Bryant, H. S., Proulx, C. M., \& Johnson, R. L. (2000). Short-term performance effects of high power, high force, or combined weight-training methods. Journal of Strength and Conditioning Research, 14, 14-20.

Hennessy, L., \& Kilty, J. (2001). Relationship of the stretch-shortening cycle to sprint performance in trained female athletes. Journal of Strength and Conditioning Research, 15(3), 326-331.

Hoff, J. (2005). Training and testing physical capacities for elite soccer players. Journal of Sports Sciences, 23(6), 573-582.

Little, T., \& Williams, A. G. (2005). Specificity of acceleration, maximum speed, and agility in professional soccer players. Journal of Strength and Conditioning Research, 19(1), 76-78.

Malina, R. M., Eisenmann, J. C., Cumming, S. P., Ribeiro, B., \& Aroso, J. (2004). Maturityassociated variation in the growth and functional capacities of youth football (soccer) players 13-15 years. European Journal of Applied Physiology, 91(5/6), 555-562. 
Markovic, G., Dizdar, D., Jukic, I., \& Cardinale, M. (2004). Reliability and factorial validity of squat and countermovement jump tests. Journal of Strength and Conditioning Research, 18(3), 551555.

Markovic, G., Jukic, I., Milanovic, D., \& Metikos, D. (2007). Effects of sprint and plyometric training on muscle function and athletic performance. Journal of Strength and Conditioning Research, 21 (2), 543-549.

Marques, M. C., Travassos, B., \& Almeida, R. (2010). A força explosiva, velocidade e capacidades motoras específicas em futebolistas juniores amadores: Um estudo correlacional. Motricidade, 6(3), 5-12.

Mourão, P., \& Gonçalves, F. (2008). A utilização dos membros superiores nos saltos verticais: Estudo comparativo entre um salto sem contramovimento sem a utilização dos membros superiores e um salto sem contra-movimento com a utilização dos membros superiores. Motricidade, 4(4), 23-28.

Paradisis, G. P., Tziortzis, S., Zacharogiannis, E., Smirniotou, A., \& Karatzanos, L. (2005). Correlation of the running-based anaerobic sprint test (RAST) and performance on the $100 \mathrm{~m}, 200 \mathrm{~m}$ and $400 \mathrm{~m}$ distance tests. Journal of Human Movement Studies, 49, 77-92.

Pellegrinotti, I. L., Daniel, J. F., Cielo, F. B. L., Cavaglieri, C. R., Neto, J. B., Montebelo, M. I. L., \& Cesar, M. C. (2008). Análise da potência anaeróbia de jogadores de futebol de três categorias, por meio do "teste de velocidade para potência anaeróbia” (TVPA) do RunningBased Anaerobic Sprint Test (RAST). Arquivos em Movimento, 4(2), 3-15.

Peterson, M. D., Alvar, B. A., \& Rhea, M. R. (2006). The contribution of maximal force production to explosive movement among young collegiate athletes. Journal of Strength and Conditioning Research, 20(4), 867-873.

Rebelo, A. N., \& Oliveira, J. (2006). Relação entre a velocidade, a agilidade e a potência muscular de futebolistas profissionais. Revista Portuguesa de Ciências do Desporto, 6(3), 342-348.

Reilly, T., Bangsbo, J., \& Franks, A. (2000). Anthropometric and physiological predispositions for elite soccer. Journal of Sports Sciences, 18(9), 669683.

Roseguini, A. Z., Silva, A. S. R., \& Gobatto, C. A. (2008). Determinações e relações dos parâmetros anaeróbios do RAST, do limiar anaeróbio e da resposta lactacidemica obtida no inicio, no intervalo e ao final de uma partida oficial de handebol. Revista Brasileira de Medicina do Esporte, 14(1), 46-50.

Sayers, S. P., Harackiewicz, D. V., \& Harman, E. A. (1999). Cross-validation of three jump power equations. Medicine $\mathcal{E}$ Science in Sports $\mathcal{E}$ Exercise, $31(4)$, 572-577.

Secora, C. A., Latin, R. W., Berg, K. E., Noble, J. M. (2004). Comparison of physical and performance characteristics of NCAA Division I football players: 1987 and 2000. Journal of Strength and Conditioning Research, 18(2), 286-291.

Sherar, L. B., Baxter-Jones, A. B. G., Faulkner, R. A., \& Russel, K. W. (2007). Do physical maturity and birth date predict talent in male youth ice hockey players? Journal of Sports Sciences, 25(8), 879-886.

Stølen, T., Chamari, K., Castagna, C., \& Wisløff, U. (2005). Physiology of soccer: An update. Sports Medicine, 35(6), 501-536.

Vescovi, J. D., \& McGuigan, M. R. (2008). Relationships between sprinting, agility, and jump ability in female athletes. Journal of Sports Sciences, 26(1), 97-107.

Vescovi, J. D., Rupf, R., Brown, T. D., \& Marques, M. C. (2011). Physical performance characteristics of high-level female soccer players 12-21 years of age. Scandinavian Journal of Medicine and Science in Sports, 21 (5), 670-678.

Wisløff, U., Castagna, C., Helgerud, J., Jones, R., \& Hoff, J. (2004). Strong correlation of maximal squat strength with sprint performance and vertical jump height in elite soccer players. British Journal of Sports Medicine, 38(3), 285-288.

(c) EY-No Todo o conteúdo da revista Motricidade está licenciado sob a Creative Commons, excepto quando especificado em contrário e nos conteúdos retirados de outras fontes bibliográficas. 